\title{
AN ADVANTAGE FOR THE EVOLUTION OF MALE HAPLOIDY AND SYSTEMS WITH SIMILAR GENETIC TRANSMISSION
}

\author{
J. J. BULL \\ Genetics Loborotory, University of Wisconsin, Modison, WI 53706, U.S.A.
}

Received 29.iii.79

\section{SUMmary}

In arthropods and rotifers a variety of genetic systems share the common property that males transmit only their mother's genome while females transmit genomes of both parents. Many of these have long been recognised because of the haploidy of males, but there are also some species in which males are diploid and yet transmit only the maternal genome. It is shown that the evolution of these maternal-genome-transmitting males (" haploid " males) is governed by the simple selective principle that maternal alleles occur at twice the frequency in gametes of haploid sons as in gametes of diploid sons. Under a simple constraint on the fitness of haploid males, selection favours mothers which produce haploid sons merely because they transmit maternal alleles at a greater rate than do diploid sons. The advantage to mothers in producing haploid sons is at the expense of the paternal genome, which is not transmitted by haploid sons. There is therefore counter-selection on fathers to produce diploid sons, and if the right genetic variation arises this countering selection will revert male haploidy to diploidy. These arguments apply to large, randommating populations but not necessarily to inbred populations.

\section{INTRODUCTION}

IN most dioecious species of animals, both sexes are diploid and transmit their maternal and paternal genes in equal frequency. In some organisms, however, males transmit only the maternal genome while females transmit genes inherited from both parents. In many of these species the male is actually haploid and the term " male haploidy" has been applied collectively to describe them, but there are also species in which males are diploid and yet transmit only the maternal genome. Examples of these unusual genetic systems may be classified into the following types (Brown, 1964; fig. 1): (i) Arrhenotoky, or haplodiploidy, in which males arise from unfertilised eggs and females arise from fertilised eggs; (ii) Paternal genome loss (my term), in which both sexes arise from fertilised eggs but either $(a)$ the paternal chromosomes are cast out of cleavage nuclei in males, leaving all tissues haploid, or $(b)$ paternal chromosomes are retained in somatic tissues but are eliminated in spermatogenesis or spermiogenesis. Arrhenotoky is thought to have arisen on several separate occasions (at least eight, Oliver, 1971; White, 1973) and is found in bees and wasps, thrips, sawflies, ticks and mites, bark beetles, scale insects, as well as some rotifers (Schrader and Hughes-Schrader, 1931; Whiting, 1945; Hartl and Brown, 1970; Nur, 1971; Oliver, 1971, 1977; White, 1973). Paternal genome loss (type $a$ ) is known in diaspidid scale insects and (type $b$ ) in lecanoid scales and sciarid flies (Metz, 1938; Hughes-Schrader, 1948; Brown, 1964; White, 1973). 


\section{Arrhenotoky}

\section{Paternal Genome Loss}

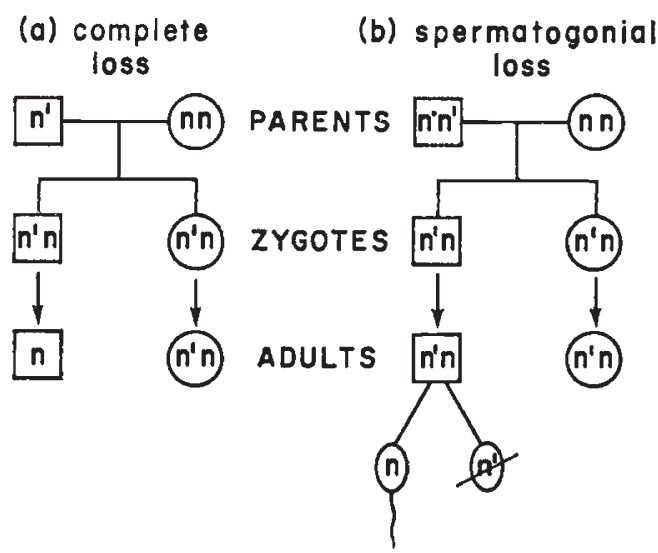

FIG. 1.-Genetic systems in which males (squares) transmit only the maternal genome and females (circles) transmit genomes of both parents. (1) Arrhenotoky, or haplo-diploidy, in which males arise from unfertilised eggs, females arise from fertilised eggs. (2) Paternal Genome Loss, in which both sexes arise from fertilised eggs, but males eliminate the paternal genome either (a) during cleavage, so that the entire male is haploid, or (b) only in the germ line, so that the male is somatically diploid but transmits as a haploid. The letters $n^{\prime}$ and $n$ distinguish genomes of the mother and father in the pedigree (but not when extrapolated to previous generations); $n$. in Paternal Genome Loss (b) indicates the paternal genome in the father, which is not transmitted to his offspring.

The above references include many excellent discussions of the evolution of arrhenotoky and paternal genome loss. It is generally thought that these genetic systems have arisen from normal diploid ones, but that the evolutionary transition is hampered by major difficulties such as low viability of haploids and complications in spermatogenesis. This naturally prompts the question of why arrhenotoky or paternal genome loss arises, if so many hurdles stand in the way. Only two quantitative studies consider this problem. Brown (1964) has shown that the evolution of paternal genome loss requires that the allele causing chromosome elimination act only when inherited from the mother; if the allele acts only when inherited from the father, diploidy is favoured. Hartl and Brown (1970, following Brown, 1964) show that arrhenotoky is selected for if the proportion of unfertilised eggs is high enough relative to the fitness of haploid males. Here the problem of the origin of arrhenotoky and paternal genome loss is considered further. My treatment rests heavily on the foundation provided by Brown and by Hartl and Brown, but their results are extended, and it is shown here that a single principle unifies the evolution of arrhenotoky and paternal genome loss. Contrary to previous ideas, it is suggested that inbreeding may complicate rather than facilitate this evolution.

\section{Definition OF TERMS: MALE HAPLOIDY AND DIPLOIDY}

The text below requires a word which collectively describes all cases of arrhenotoky and paternal genome loss, but the literature on these genetic systems lacks such a word. Previously, the term "male haploidy" has been applied to many of these systems, but only to those in which the male is 
haploid (at least, haploid in the germ line, Haldane, 1957; White, 1973). Here that meaning is extended: Male haploidy indicates any genetic system in which males transmit only the maternal genome; the maternal-genometransmitting males are referred to as haploid males, regardless of their ploidy. In essence, they transmit as if they are haploid. Diploidy, therefore, indicates the more normal state in which males as well as females are diploid and transmit genes of both parents (diploid males). These terms clearly leave something to be desired, and it is not intended that the extended definitions be adopted outside of this paper. My only alternative would be to propose two new terms, whose unfamiliarity to readers might disrupt the flow of the presentation.

\section{The advantage of male haploidy}

The advantage of male haploidy can be thought of as operating at the individual level over a two-generation period (from $P_{1}$ to $F_{2}$ ). The basis for the argument is that haploid sons when compared to diploid sons differentially contribute their $\left(\mathrm{P}_{1}\right)$ maternal alleles to the $\mathrm{F}_{2}$ generation. There are two components to this differential contribution. The first component is that maternal alleles are twice as frequent in gametes of haploid sons as in gametes of diploid sons (fig. 2). The two-fold difference is due to the segregation of paternal alleles to the sperm of diploid males, which dilutes the maternal alleles by 50 per cent. The second component of the argument is a possible lower fitness of haploid sons compared to diploid sons. If the relative fitness of haploid sons is denoted $s$, then a haploid son contributes $2 s$ maternal alleles to the $F_{2}$ generation for every maternal allele contributed by a diploid son. Thus, if $s>1 / 2$, a $\mathbf{P}_{1}$ female realises a greater contribution to the $F_{2}$ generation if she produces haploid sons rather than diploid sons.

This principle may be the basis for the evolution of male haploidy. If $s>1 / 2$, then genes which act in the mother to substitute haploid sons in place of diploid sons are favoured. A maternal effect restriction is placed on this

\section{Pedigree with Diploid Sons}

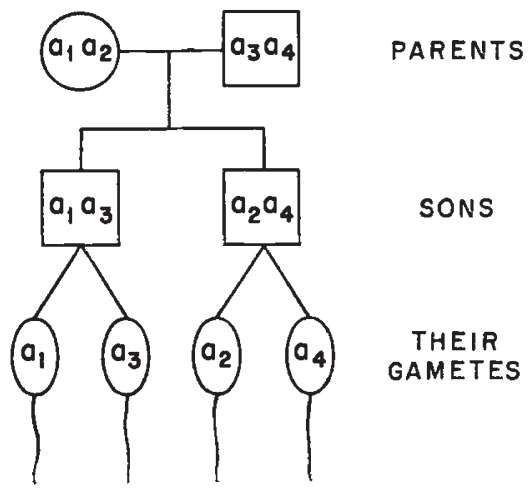

\section{Pedigree with} Haploid Sons 
process because genes which act in the father to produce haploid sons eliminate themselves and therefore should not be favoured. And once male haploidy has arisen, genes which act in the father to restore diploidy will be favoured and select against male haploidy. Therefore, the origin of male haploidy requires mutations which act in the mother to produce haploid sons, and the maintenance of male haploidy requires that these maternal processes not be vulnerable to mutations which act in the father to restore diploid sons. As far as these arguments are concerned, it is seen that the somatic ploidy of the male is irrelevant, and the important distinction is whether or not he transmits the paternal genome. It is for this reason that I have defined the term " male haploidy" to cover all cases of arrhenotoky and paternal genome loss.

\section{Models: Paternal genome loss}

Some models will help illustrate the hypothesised advantage for male haploidy. The first models will consider the evolution of paternal genome loss in a population with diploid males. The genetic control of chromosome loss is not understood in any male haploid species, so the model will assume the simplest possible genetic control. Since the only clear-cut difference between diploid and haploid males is the loss of paternal chromosomes in the latter, the model will assume that a single locus is responsible for the difference. In the origin of male haploidy, it is likely that the normal physiological processes in the species cause spermatogenesis to segregate paternal and maternal alleles to sperm. Let mutation $A$ arise in this population, and let $A$ cause the loss of paternal chromosomes in some males: specifically, if a male inherits $A$ from his father, then $A$ has no effect, but if $A$ is inherited from his mother, then $A$ causes the paternal genome to be eliminated. This formulation is consistent with the aforementioned concept of a gene which acts in the mother to substitute haploid sons for diploid sons. All other alleles at this locus are collectively labelled $a$ and have no effect on spermatogenesis. Haploid males are assigned fitness $s$ relative to diploid males. There is, however, no distinction in this model between haploid males and males which eliminate just the paternal allele at this locus.

In addition, the sex determining mechanism must be specified. Female heterogamety will be assumed ( $X Y$ females, $X X$ males). The alternative of male heterogamety ( $X Y$ or $X O$ males) precludes paternal genome loss throughout the population because the haploid males transmit only the $X$ chromosome and thereby produce all daughters (Brown, 1964). Female heterogamety avoids this problem so that the sex ratio is unaffected. Female heterogamety is, in fact, known in Sciara (Metz, 1938). To simplify the algebra, there will be no linkage between the sex chromosomes and the locus of $A$. This specification leads to a population with the following genotypes.

$\begin{array}{ccclc}\begin{array}{c}\text { Frequency } \\ \text { in zygotes }\end{array} & \text { Male } & \text { Fitness } & \text { Female } & \begin{array}{c}\text { Frequency } \\ \text { in zygotes }\end{array} \\ m_{1} & \text { aaXX } & 1 & a a X Y & f_{1} \\ m_{2} & \operatorname{AaXX} & 1 & \operatorname{AaXY} & f_{2} \\ m_{3} & A(-) X(X) & s & A A X Y & f_{3} \\ & & \sum_{i} m_{i}=\sum_{i} f_{i}=1 & & \end{array}$


In the haploid male genotype $A(-) X(X)$, the brackets, ( ), represent the possible somatic existence of the paternal allele $A$ or $a$ and the paternal $X$ chromosome, which are not transmitted. Note that the diploid male genotype $A A X X$ is not produced, because every male inheriting $A$ from his mother becomes haploid. Also, the haploid male genotype $a(-) X(X)$ is absent because no male that inherits $a$ from his mother is haploid. If a different model of gene action had been specified, either of these two male genotypes might have been present.

Under random mating with discrete generations, infinite population size, Mendelian segregation except for haploid males, and enumeration of genotype frequencies before fitness effects, the recursion formulae follow. Letting $W \cdot x=m_{1}+0.5 m_{2}, y=f_{1}+0.5 f_{2}, W=m_{1}+m_{2}+s \cdot m_{3}$

$$
\begin{array}{ll}
m_{1}^{\prime}=x y & f_{1}^{\prime}=x y \\
m_{2}^{\prime}=y(1-x) & f_{2}^{\prime}=x(1-y)+y(1-x) \\
m_{3}^{\prime}=(1-y) & f_{3}^{\prime}=(1-x)(1-y)
\end{array}
$$

or,

$$
x^{\prime}=\frac{0 \cdot 5 y(1+x)}{y+s(1-y)}, \quad y^{\prime}=\frac{x+y}{2}
$$

At equilibrium, (la) indicates that $x=y$ and that

$$
x(1-x)\left(s-\frac{1}{2}\right)=0
$$

In words, only complete male haploidy or complete diploidy is equilibrial unless the fitness of haploid males is $1 / 2$. If $s=1 / 2$, then the frequency of haploid males can take on any value from 0 to 1 at equilibrium. Stability analysis indicates that male haploidy evolves $(x, y \rightarrow 0)$ if $s>1 / 2$ but is selected against $(x, y \rightarrow 1)$ if $s<1 / 2$ (Appendix). Although male haploidy is favoured for all $s>1 / 2$, the strength of selection increases as $s$ increases. The parameter $s$ combines viability, mating ability, and the proportion of euploid gametes.

The plausibility of mutations for paternal genome loss is not unreasonable. First, there are various means of creating a mechanism of paternal genome loss, as shown by scale insects and Sciara. In Sciara, males are diploid (biparental) in all tissues, and spermatogenesis dispenses with the paternal genome (Metz, 1938). This also occurs in lecanoid scales, although the paternal genome is heterochromatised and inactivated in most tissues (Brown, 1964). In diaspidid scales, males are actually haploid because the paternal genome is eliminated from cleavage nuclei (Brown, 1964). Another possible but unknown male haploid system is pseudogamy, in which the sperm triggers embryogenesis but degenerates after entering eggs destined to become males. In the context of model (1), all these paternal genome loss mechanisms require (i) a maternal effect mutant, (ii) a basis for distinguishing paternal and maternal chromosomes, and (iii) a sex-limited effect.

Although the genetics of paternal genome loss systems are not sufficiently understood to evaluate the validity of this model, a number of mutations in Drosophila possess one or more of the required characteristics, indicating that they are not unreasonable. The well-known system of SD (segregation distorter) in Drosophila is a naturally occurring mechanism of choromsome 
loss in males (Hartl and Hiraizumi, 1976). Males heterozygous for the SD loci show a marked distortion in the transmission ratio of these alleles. SD operates by preferentially destroying spermatids which inherit the unfavoured allele, regardless of its parental origin. Other mutations in Drosophila demonstrate maternal effect and paternal-maternal discrimination of chromosomes. Claret and Mitotic Loss Inducer are two recessive mutations which cause chromosome loss only in embryos of homozygous mothers, regardless of paternal or offspring genotype (Baker and Hall, 1976; Hall et al., 1976). The former leads to a preferential loss of one or more maternal chromosomes during cleavage, while the latter mutation indiscriminately causes loss of paternal and maternal chromosomes later than cleavage. An especially interesting mutation described by Levitan (1963) showed maternalpaternal discrimination by causing breakage of paternal chromosomes in offspring of mutant mothers. It is not clear whether the mutation showed a maternal effect or was simply inherited cytoplasmically (Levitan and Williamson, 1965). A type of maternal-paternal discrimination of chromosomes is also evident at the gonomeric first cleavage division in Drosophila. The maternal and paternal pronuclei do not fuse, but lie adjacent until the first telophase unites them in each daughter nucleus (Sonnenblick, 1950). This system would seem particularly susceptible to modification, so that the paternal genome could be eliminated at the outset. Therefore, while no mutations in Drosophila are known to fulfil all of the requirements for paternal genome loss (as in 1), the evidence suggests that the required mutations occur in some species.

The selective advantage of male haploidy when $s>1 / 2$ is not restricted to the specifics of model (1). With female heterogamety, the equilibrial conditions of ( $\mathrm{lb}$ ) are also generated if a recessive or dominant allele $A$ acts in the mother to cause paternal genome loss in all her sons. With male heterogamety, fixation of allele $A$ is not possible, but whenever $s>1 / 2, A$ can invade the population and increase to a stable polymorphism. This polymorphism can be invaded by an allele which converts the sex determining mechanism to female heterogamety (cf. Bull and Charnov, 1977).

Brown (1964) studied a model for the evolution of paternal genome loss. Instead of assuming two distinct types of males in the population, Brown assumed that there were two types of spermatogonia in males, (i) spermatogonia that eliminated the paternal genome, and (ii) diploid spermatogonia. He then studied selection on a gene $(A)$ which changed the proportion of the two types of spermatogonia in males. He observed that selection favoured male haploidy if $A$ acted only when inherited from the mother or that evolution proceeded toward diploidy if $A$ acted only when inherited from the father. In the context of the model (1) above, Brown assumed $s=1$, because meiosis was assumed to be perfect in both kinds of spermatogonia. Therefore, Brown's first result is consistent with the treatment here.

The advantage realised by maternal alleles in haploid males is at the expense of paternal alleles. Therefore, the evolution and maintenance of male haploidy should be considerably different if a paternally derived $A$ acts to eliminate the paternal genome, as foreshadowed by Brown. Two variations of paternal action are possible: allele $A$ causes paternal genome loss in males regardless of its parental origin (model 2), or allele $A$ causes paternal genome loss only when inherited from the father (model 3 ). The genotypes corresponding to each of these models follow. 
(2)

\begin{tabular}{|c|c|c|c|c|c|c|c|c|c|}
\hline Freq. & Male & Fitness & Female & Freq. & Freq. & Male & Fitness & Female & Freq. \\
\hline$m_{1}$ & $a a X X$ & 1 & $a a X Y$ & $f_{1}$ & $m_{1}$ & $a a X X$ & 1 & $a a X r$ & $f_{1}$ \\
\hline$m_{2}$ & $a(A) X(X)$ & $s$ & $A a X Y$ & $f_{2}$ & $m_{2}$ & $\operatorname{AaXX}$ & 1 & $\operatorname{AaXY}$ & $f_{2}$ \\
\hline \multirow[t]{2}{*}{$m_{3}$} & $A(-) X(X)$ & $s$ & $A A X \Upsilon$ & $f_{3}$ & $m_{3}$ & $a(A) X(X)$ & $s$ & $A A X Y$ & $f_{3}$ \\
\hline & & & & & $m_{4}$ & $A(A) X(X)$ & $s$ & & \\
\hline
\end{tabular}

The possible genotypes are again a consequence of the mode of action of $A$. In both (2) and (3), haploid males can be either $a$ or $A$ because only the maternal allele is retained if $A$ enters the zygote with the sperm.

Recursion formulae in (2) are, for $W \cdot x=m_{1}+s \cdot m_{2}, y=f_{1}+0 \cdot 5 f_{2}$, $W=m_{1}+s \cdot\left(1-m_{1}\right)$

$$
\begin{array}{ll}
m_{1}^{\prime}=x y & f_{1}^{\prime}=x y \\
m_{2}^{\prime}=y(1-x) & f_{2}^{\prime}=x(1-y)+y(1-x) \\
m_{3}^{\prime}=(1-y) & f_{3}^{\prime}=(1-x)(1-y)
\end{array}
$$

leading to $x^{2}(1-x)(s-1)=0$ at equilibrium. Stability analysis and numerical studies (Appendix) suggest that male haploidy evolves if $s>1$, diploidy evolves if $s<1$, and both haploid and diploid males may coexist at equilibrium if all males have the same fitness. This model, recall, represents gene $A$ as causing paternal genome loss in all males, independently of the parental origin of $A$. Intuitively, this situation can be compared to an autosomal gene in a diploid which affects the fitness of one sex. Since $A$ is transmitted from father to son approximately as often as from mother to son (if $s$ is near unity), allele $A$ eliminates itself and gives an advantage to $a$ in haploid males approximately as often as $A$ eliminates $a$ and gives itself the advantage. Therefore, the gametes of haploid males transmit $A$ in approximately the same frequency that occurred in them as zygotes, and the only basis for selection is the fitness $s$ of the haploid males.

Model (3) is especially interesting. Allele $A$ preferentially causes its own loss whenever it is transmitted through sperm to a male. Recursion formulae are, for $W \cdot x=m_{1}+0 \cdot 5 m_{2}+s \cdot m_{3}, y=f_{1}+0 \cdot 5 f_{2}, W=m_{1}+m_{2}+s \cdot\left(m_{3}+m_{4}\right)$

$$
\begin{array}{ll}
m_{1}^{\prime}=x y & f_{1}^{\prime}=x y \\
m_{2}^{\prime}=x(1-y) & f_{2}^{\prime}=x(1-y)+y(1-x) \\
m_{3}^{\prime}=y(1-x) & f_{3}^{\prime}=(1-x)(1-y) \\
m_{4}^{\prime}=(1-x)(1-y) &
\end{array}
$$

These lead to $x(1-x)=0$ at equilibrium. That is, there is no finite value of $s$ for which haploid and diploid males may coexist at equilibrium. The stability analysis and numerical studies suggest that diploidy always evolves (Appendix). This result therefore agrees with Brown's second result for $s=1$. A particularly interesting interpretation of (3) is that reversion to diploidy is favoured no matter how low the fitness of diploid sons. One can therefore imagine a population evolving in a circuitous route from diploidy to male haploidy, and again to diploidy, with the males in the third step being of such low fitness as to endanger the survival of the population. 
In conclusion, the three models present an interesting picture for the evolution of male haploidy via a gene which causes the loss of paternal chromosomes in males (fig. 3). (1) If the paternal chromosomes are eliminated by $A$ only when $A$ is of maternal origin, then the haploid males need to have a fitness greater than half that of the diploid males $(s>1 / 2)$. (2) If allele $A$ causes the loss of the paternal genome in all male zygotes, the haploid males must have greater fitness than the diploid males $(s>1)$. (3) If allele $A$ causes the loss of paternal chromosomes only when $A$ is of paternal origin, then male haploidy can never evolve. Alternatively, in order for male haploidy to revert to diploidy (via allele $a$ ), the relative fitness of the diploids $(1 / s)$ must exceed 2 if $a$ acts to restore diploidy only when of maternal origin, must exceed unity if $a$ acts independently of parental origin, but must merely exceed zero if $a$ acts only when of paternal origin.

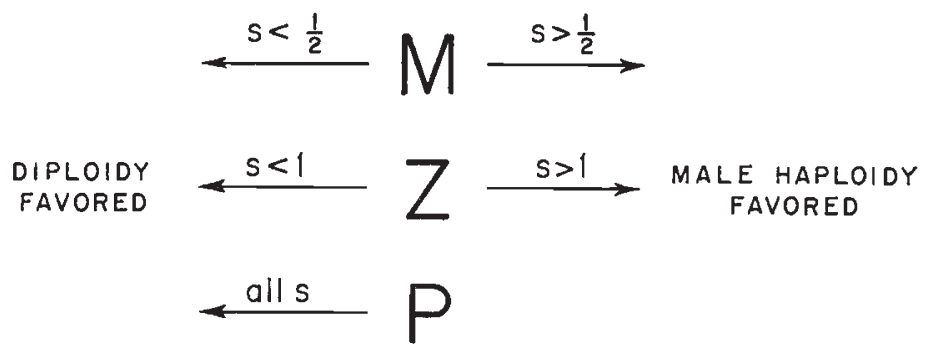

FiG. 3.-Diagrammatic summary of the three models for Paternal Genome Loss. If a gene causing paternal chromosome elimination in males does so only when inherited from the mother ( $M$, top line), then the fitness of haploid sons $(s)$ need only be half that of the diploid sons in order for male haploidy to evolve. If the elimination occurs in all male zygotes inheriting the gene ( $Z$, middle), the haploid males must have greater fitness than diploid males. If the gene acts only when inherited paternally, $(P$, bottom line), male haploidy can never evolve.

Male haploidy is therefore most likely to evolve if the genes controlling paternal genome loss act only when inherited from the mother. Male haploidy is exceptionally prone to revert to diploidy if mutations arise which prevent themselves from being eliminated in males when they are transmitted through sperm. These models indicate that paternal genome loss systems may evolve in a seesaw fashion between male haploidy and diploidy, depending upon the relative rates of incorporation of different mutations. This conclusion was reached by Brown (1964) under the constraint $s=1$. Brown further suggested that four types of genetic systems in scale insects represented this evolutionary oscillation between male control and female control of paternal genome loss. The primitive scales are diploids. In lecanoid scales the paternal genome is present, but is not transmitted through sperm. In the Comstockiella system, only one paternal chromosome is eliminated (different spermatocytes eliminating a different paternal chromosome), and a maternal chromosome is duplicated to fill the vacancy. Comstockiella, therefore, represents nearly a diploid condition. The fourth scale system, of diaspidids, again eliminates the entire paternal genome, but in this the paternal genome is eliminated at cleavage. Brown notes that there is no selection for the change from lecanoid to diaspidid since all paternal chromosomes are eliminated in both systems. He proposed that the lecanoid system arose from the primitive diploids, the Comstockiella system 
arose from the lecanoid, and the diaspidid then arose from the Comstockiella system. Each step in this process is an alternation from diploidy to male haploidy or the reverse. Taxonomic relationships of scale insects support this theory (Brown, 1964).

In order for male haploidy to evolve, the fitness requirements on haploid males must be satisfied immediately, when $A$ first appears: if haploid males are not sufficiently fit at the outset, they will never become common enough nor persist long enough for selection to improve their fitness. Therefore, the paternal genome loss systems in scale insects and sciarid flies must have been considerably co-ordinated in meiosis and permitted high viability of the haploid males when they first arose.

\section{MODELS: ARRHENOTOKY}

The evolution of arrhenotoky from diploidy is thought to occur in a manner different from the above (Hartl and Brown, 1970). Two prerequisites seem necessary: (i) facultative parthenogenesis, the ability to produce fertile offspring from eggs that go unfertilised, and (ii) a sex determining mechanism causing the haploids to be male. A species with these capacities should then have a possibility of becoming arrhenotokous (Hartl and Brown, 1970, superseding Brown, 1964). Hartl and Brown supposed that arrhenotoky has a diploid ancestry which already possesses the appropriate sex-determining mechanism. This assumption is valid in so far as it can be assumed that at least some diploid species have such a sex-determining mechanism, which is certainly plausible in view of the variety of sexdetermining mechanisms in arthropods. They proceed to consider the evolution of parthenogenesis. Hartl and Brown suggest that selection favours facultative parthenogenesis in populations which experience unavoidable shortages of sperm to fertilise eggs. The unfertilised eggs are otherwise wasted, so that any genes which cause development of these eggs are favoured. It is important here to distinguish between genes for facultative parthenogenesis and those for obligate parthenogenesis. Facultative parthenogenesis assumes that all eggs await fertilisation, but then develop even if unfertilised. Therefore, facultative parthenogenesis can evolve without upsetting the normal process of sexual reproduction in the population. Hartl and Brown, however, point out that many species may have a latent ability to be parthenogenetic, since unfertilised eggs can often be induced artificially to begin development (Whiting, 1945). Therefore the advent of some new environmental stimulus might be enough to generate facultative parthenogenesis.

Once facultative parthenogenesis has evolved, haploid males are present as a consequence of unfertilised eggs. Diploid males may or may not be present, depending upon the fitness of haploid males $(s)$ and the proportion of unfertilised eggs $(u)$ in the population. That is, even though the population consists entirely of females capable of producing sons parthenogenetically, diploid males are not necessarily eliminated. Conversely, diploid males are not immune to loss. Following Hartl and Brown, if sex is determined according to

\begin{tabular}{lllc} 
& \multicolumn{2}{c}{ Male } & Female \\
Genotype & $X Y$ & $X$ & $X X$ \\
Freq. & $p$ & $1-p$ & 1 \\
Fitness & 1 & $s$ & 1
\end{tabular}


and all matings yield an average of $1-u$ fertilised eggs, $u$ unfertilised ones, then,

$$
W \cdot p^{\prime}=\frac{p(1-u)}{2}, \quad W=u s+\frac{p(1-u)}{2}
$$

for discrete generations, random mating, and infinite population size. At equilibrium

$$
\hat{p}=\frac{1-u-2 u s}{1-u}, \text { or zero }
$$

Hartl and Brown showed that this equilibrium is always approached, so that if $1-u-2 u s \leqq 0$, diploid males are lost, and full arrhenotoky evolves.

The Hartl-Brown result shows that for $s>0$, haploid males can completely replace the diploid males if $u$ becomes large enough. Therefore, $u$ ultimately controls the evolution of arrhenotoky. Hartl and Brown treat $u$ as a constant, assuming it to be a consequence of demographic conditions. Thus, it was hard to imagine diploid males being lost completely unless haploid males were highly fit, because unrealistically high values of $u$ are required to compensate for low fitness. For the present discussion, however, it is useful to consider how selection acts on $u$, since this parameter controls the ratio of haploid to diploid males in a brood. Clearly, selection always favours the male to minimise $u$ in his matings (subject to limitations on sperm production) because he contributes nothing to his mate's unfertilised eggs (cf. Hamilton, 1967). In the female, selection on $u$ depends upon the fitnesses of haploid and diploid sons, as outlined above. Since the female is likely to have the final control over the fertilisation of her eggs, the biologically relevant problem in the evolution of arrhenotoky is the evolution of fertilisation $(u)$ as controlled by the female.

The problem can be addressed formally with a model that superimposes autosomal control of $u$ on the Hartl-Brown model of arrhenotoky. Let $A$ be a dominant modifier of $u$, controlling fertilisation in the female, such that $A A$ and $A a$ females fertilise $1-u^{*}$ of their eggs, while $a a$ females fertilise $1-u$ of them. To simplify the algebra, $A$ is considered only when rare. The following genotypes are of interest.

\begin{tabular}{lrlrc} 
Male & \multicolumn{1}{c}{ Freq. } & Female & Freq. & Level of fert. \\
$a a X Y$ & $p-\varepsilon_{1}$ & $a a X X$ & $1-\varepsilon_{3}$ & $1-u$ \\
$A a X Y$ & $\varepsilon_{1}$ & $A a X X$ & $\varepsilon_{3}$ & $1-u^{*}$ \\
$a X$ & $1-p-\varepsilon_{2}$ & $A A X X$ & 0 & $1-u^{*}$ \\
$A X$ & $\varepsilon_{2}$ & & & \\
$A A X Y$ & 0 & & &
\end{tabular}

With low frequencies of $A$, the terms $p-\varepsilon_{1}, 1-p-\varepsilon_{2}$, and $1-\varepsilon_{3}$ are approximated by omitting the $\varepsilon_{i}$. Also, second order terms in $\varepsilon$ (i.e., genotypes involving $A A$ ) are ignored. Assuming random mating, Mendelian segregation, and that the population frequency of diploid males is at the equilibrium $(\hat{p})$ given by equation $(4 \mathrm{~b})$, the recursion formulae are

$$
\varepsilon_{1}^{\prime}=\frac{\varepsilon_{1} \frac{1-u}{4}+\hat{p} \varepsilon_{3} \frac{1-u^{*}}{4}}{W_{m}}
$$




$$
\begin{gathered}
\varepsilon_{2}^{\prime}=\varepsilon_{3} \frac{u^{*} s}{2 W_{m}} \\
\varepsilon_{3}^{\prime}=\frac{(1-u)\left(\frac{\varepsilon_{1}}{4}+\varepsilon_{2}\right)+\varepsilon_{3} \frac{1-u^{*}}{2}\left(1-\frac{\hat{p}}{2}\right)}{W_{f}} \\
W_{m}=\frac{1-u}{2}, \quad W_{f}=\frac{1-u}{2}+u s
\end{gathered}
$$

The characteristic equation for this system is

$$
\begin{array}{r}
F(\lambda)=\lambda^{3}-\lambda^{2}\left[\frac{1}{2}+\frac{1-u^{*}}{2(1-u)}\right]-\lambda\left[\frac{\left(1-u^{*}\right)(1-u-2 u s)}{4(1-u)(1-u+2 u s)}-\frac{1-u^{*}}{4(1-u)}\right. \\
\left.\quad+\frac{2 u^{*} s}{1-u+2 u s}\right]+\frac{u^{*} s}{1-u+2 u s}=0
\end{array}
$$

Rather than solve $F(\lambda)$, it is sufficient to note the behaviour about $\lambda=1,0$. $F(\lambda)$ has three roots. Since $F(0)$ is positive and $F(-\infty)$ is negative, there must be at least one negative real root. At $\lambda=1$,

$$
F(1)=\frac{(2 s-1)\left(u-u^{*}\right)}{2(1-u+2 u s)}
$$

If $F(1)$ is less than zero, then there must be one real root between zero and unity and one real root greater than unity, since $F(\infty)$ is positive. This information is sufficient to demonstrate that selection favours (i) increasing $u$ if $s>1 / 2$ or (ii) decreasing $u$ if $s<1 / 2$, since these two combinations satisfy $F(1)$ less than zero. Since a change in $u$ causes a corresponding change in the frequency of haploid males, selection thereby favours arrhenotoky if $s>1 / 2$, while selection favours diploidy if $s<1 / 2$. Although this analytical treatment deals only with rare modifiers, numerical studies of this model show that if $s>1 / 2$, selection favours arrhenotoky at intermediate gene frequencies as well.

These equations are valid until diploid males are lost. At this point, $1-u-2 u s=0$, leading to $2 s-1=\frac{1-2 u}{u}$, and

$$
F(1)=\frac{(1-2 u)\left(u-u^{*}\right)}{4 u(1-u)}
$$

Therefore, a sex ratio of $1 / 2$ is evolutionarily stable (an ESS of Maynard Smith, 1978), because it can never be invaded by a different sex ratio, and selection of rare modifiers favours a sex ratio closer to $1 / 2$. This corresponds to Hamilton's (1967) and Hartl and Brown's result of a sex ratio equilibrium of $1 / 2$ in outbred arrhenotokous populations.

A point of interest is that the sex-determining mechanism in the ancestral population is assumed to be male heterogamety. Haploids would be expected to be female if sex was determined by either an $X$ /autosome balance or a dominant- $Y$ mechanism (e.g., White, 1973), so there may be only a special set of male heterogametic systems which are consistent with the model. 
Male heterogamety is often considered the immediate ancestor of arrhenotoky, because it occurs in the closest diploid relatives of some arrhenotokous species as well as in the majority of diploid arthropods (Whiting, 1945; Brown, 1964; Hartl and Brown, 1970; White, 1973; Kerr, 1974). In many of the arrhenotokous Hymenoptera, however, the sex-determining mechanism would operate as female heterogamety in a diploid population, because females are heterogametic and males homogametic (diploid homozygotes as well as haploids are male, Whiting, 1945). The above model does not apply to the hymenopteran case, but the same principle of $s>1 / 2$ again governs the evolution to arrhenotoky (Bull, to be published).

To conclude, arrhenotoky differs from paternal genome loss because its evolution is a two-step process. The first step is the evolution of facultative parthenogenesis, with the unfertilised eggs developing into males. Facultative parthenogenesis can be selected for merely as a consequence of some eggs being unfertilised, but may result fortuitously from some environmental stimulus. This may be a difficult step because it is doubtful if many populations have the genetic variation to allow unfertilised eggs to develop as well as a sex-determining mechanism that causes them to be male (Whiting, 1945; Hartl and Brown, 1970). However, it is appealing in that the advantage of facultative parthenogenesis may apply to many populations and that such genes can accumulate even though the fitness of haploids be much less than $1 / 2$. The second step is the replacement of diploid males by haploid males. This occurs if $1-u-2 u s \leqq 0$ (Hartl and Brown, 1970). If $s<1 / 2$, then selection favours the female to minimise $u$, and it seems unlikely that arrhenotoky will evolve. Continued selection upon haploid males may improve their fitness, though, and $s$ may come to exceed $1 / 2$. Once $s>1 / 2$, selection favours the female to fertilise fewer eggs, until half the eggs in the population go unfertilised. This automatically leads to the loss of diploid males. The parallel between the evolution of paternal genome loss and arrhenotoky is thus demonstrated.

Model (3) showed that paternal genome loss male haploidy can revert to diploidy if mutations arise which act in the father to restore diploid sons. A parallel occurs in arrhenotoky. Selection favours genes acting in the father to increase the proportion of eggs fertilised without bound, even though these offspring are female (Hamilton, 1967). This does not alone lead to a reversion to diploidy, but the sex ratio bias $(u \rightarrow 0)$ lowers the restrictions on the re-invasion of diplaid males ( $1-u-2 u s>0$ for the re-invasion). A priori, it seems that the female would have enough control over the fertilisation of her eggs (through behavioural as well as anatomical means) that arrhenotoky would be secure from the re-invasion of diploidy, assuming $s>1 / 2$.

\section{INBREEDING}

One of the obvious complications in the evolution of male haploidy is that the first haploid males to arise will suffer from the expression of detrimental, recessive alleles that are otherwise masked in normal diploid males, unless males are somatically diploid and biparental as in Sciara. It has been suggested that inbred species are more apt to foster male haploidy than outbred species because inbreeding keeps recessive detrimental alleles at low frequency and should thus reduce the discrepancy in fitness between haploid and diploid males (Brown, 1964; Hartl and Brown, 1970). This argument has 
merit, but it ignores the possibility that inbreeding has detrimental effects on the evolution of male haploidy that might compensate for the increased viability of haploids. Only if inbreeding has no effect on the result $(s>1 / 2)$ can it be assumed safely that inbreeding enhances the evolution of male haploidy. There are at least two potential complications of inbreeding. One is that the advantage of male haploidy (as postulated here) is derived from converting $A a$ males into $A$ males ( $A$ of maternal origin). Inbreeding, by reducing heterozygosity, reduces the number of $A a$ zygotes and thus weakens the basis for the advantage of male haploidy. The second complication of inbreeding comes from the viability and fertility problems of haploid males. Under random mating, all genotypes of females encounter haploid males in proportion to their (female) frequencies; thus, if it is disadvantageous to mate with haploid males (because of aneuploid sperm, for example), all females suffer proportionally and there is no basis for selection among the female genotypes. Inbreeding results in preferential mating between haploid males and females with allele $A$, thus imposing a double burden on the male haploidy allele.

It is readily seen that the evolution of male haploidy (as in 1) is somewhat more restricted under complete inbreeding than under outbreeding. In a population consisting entirely of sib-mated lines, for example, the advantage of male haploidy shown for outbreeding no longer applies because virtually all sib-mated lines are homozygous. Male haploidy will have an advantage only if lines with haploid males produce more grandchildren than lines with diploid males. This requires that the haploid males produce no more aneuploid sperm than diploid males, since aneuploid sperm will reduce the number of surviving offspring. There may also be increased viability constraints on the haploid males (above $s>1 / 2$ ), although this will depend upon how female fertility varies with the number of surviving brothers. Low haploid male survival may also facilitate outcrossing, between their sisters and diploid males. It is not obvious to what extent these restrictions apply to intermediate levels of inbreeding, but it does not seem likely that there will be any unqualified facilitation of the evolution of male haploidy.

\section{Discussion}

The above models show that a simple evolutionary principle provides an advantage for the evolution of male haploidy, whether the haploidy be due to paternal genome loss or to development from unfertilised eggs (arrhenotoky). The advantage is derived from the two-fold representation of maternal alleles in gametes of haploid sons over diploid, biparental sons. Whenever the fitness of haploid males exceeds half that of diploid males, selection favours genes which act in the mother to produce haploid sons in place of diploid sons merely because maternal alleles are thereby transmitted at a greater rate. The evolution of male haploidy is not impossible, but is more restricted, if the genes controlling chromosome loss act regardless of parental origin. However, the evolution of male haploidy becomes impossible if the genes controlling chromosome loss act only when inherited from the father. Of course, a variety of interactions may occur between modifiers of these processes, and it is not clear if the conclusions from these simple models will apply directly to the compounding of genetic interactions.

The scale insects are the most promising group in which to test these 
theories, with possibilities for studying the genetics of chromosome loss in male haploidy as well as in the reversion to diploidy. The models could be refuted entirely if it was shown that chromosome loss in males is controlled only by the father. The observations most favourable for the models are the demonstrations that (i) chromosome loss in haploid males is controlled only by the mother, and (ii) chromosome retention in species reverting to diploidy is controlled by only the father. Brown (1964) correctly recognised the basic difference between maternal and paternal control of chromosome lossretention and discussed his models in the context of genetic systems in scale insects. This paper has generalised Brown's findings and extended them to consider the fitness values of haploid males which govern the evolution of and reversion of paternal genome loss male haploidy.

The advantage of male haploidy should be sufficient to cause it to displace diploidy whenever the appropriate mutations arise and the necessary prerequisites are met in the ancestral population. One must therefore agree with Whiting (1945) and Hartl and Brown (1970) that the reason male haploidy is not more common is that too few species have overcome the initial hurdle of producing the first, sufficiently fit haploid male.

White (1954, 1973) proposed and Haldane (1957) concurred that the evolution of male haploidy was merely an extreme development of sex linkage, whereby the entire genome became sex-linked and the $r$-portion degenerated and was lost completely $\left(X_{1} \ldots X_{n} 0\right.$ males $)$. This argument does not seem to carry much weight. It was noted above that male heterogamety cannot operate in paternal genome loss systems, because haploid males produce only one kind of sperm ( $X$-type), and since all eggs are fertilised there is no basis for male determination (Brown, 1964). Male heterogamety could lead to arrhenotoky, but a large sex linkage offers no short cut. Assuming that the entire genome is sex-linked in a diploid population, males would be arising from fertilised eggs even as the $Y$-portion degenerated. Arrhenotoky could not arise until parthenogenesis evolved. Thereafter, the population would contain males arising from fertilised eggs (with virtually no paternal genome) and haploid males, and arrhenotoky would become fixed only when the haploid males displaced the fertilised males. This process is the same as in (4). White (1973) may be correct that haploids are most likely to be viable in $X 0$ species with large sex linkages, but his model does not bypass any of the steps in the evolution of male haploidy as pictured above.

The selective advantage for the evolution of male haploidy stems from a two-fold representation of maternal genes in gametes of haploid sons in comparison to diploid sons. A similar principle is thought to be the basis for the evolution of all-female reproduction (parthenogenesis and gynogenesis): mothers which produce daughters parthenogenetically or gynogenetically gain a two-fold representation in these daughters because the maternal genes are transmitted to all rather than half the eggs (Williams, 1975, p. 9, after Maynard Smith, 1971). (Whether parthenogenesis or gynogenesis is indeed favoured may depend upon other circumstances, but the two-fold representation in fatherless daughters is thought by these authors to be fundamental.) In view of this parallel between male haploidy and all-female reproduction, it might be conjectured that the advantage derived from a two-fold genetic representation is a selective basis for the evolution of any genetic system in which the genes of one parent are excluded from the gametes in offspring of 
one type. Male haploidy and parthenogenesis constitute only two of the many possibilities for such systems. Biased transmission ratios may accrue to single loci or small regions rather than entire genomes, and may be a property of all heterozygotes of one or both sexes, regardless of which parent contributed each allele. The systems of SD in Drosophila (Hartl and Hiraizumi, 1976), $t$-alleles in mice (Yanagisawa et al., 1961), spore killer genes in yeast (Turner and Perkins, 1976; Turner, 1977), and gamete eliminator in tomato (Rick, 1971) illustrate some of these alternatives. The advantage of the biased segregation is realised provided the relative fitness of the " uniparental" type exceeds one-half the reciprocal of the genetic transmission fraction. For example, SD in Drosophila is selectively favoured provided males with 100 per cent distortion have more than half the fertility of normal males (Charlesworth and Hartl, 1978).

Male haploidy is unique among animals in that it is the only common genetic system with two sexes in which offspring of one sex transmit only the genome inherited from one parent. In theory, four types of these genetic systems exist:

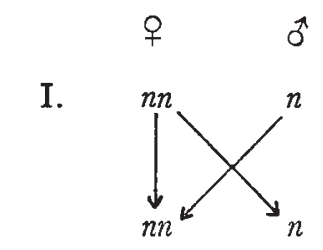

III.

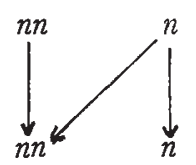

II.
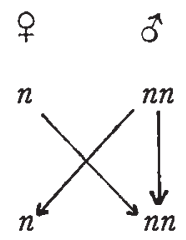

IV.

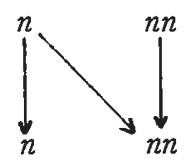

where $n$ represents the genome inherited from one parent, the arrows indicate the transmission of these genomes, and each sex is restricted to one side of the pedigree as indicated. At the outset, one might suppose that these four systems should be equally common, since the same selective principle applies to the origin of each (as above). If species with male haploidy are seemingly more common than species with any of the other three systems, then there must be some bias favouring either the evolution of, or the observation of, male haploidy. A preliminary investigation of the properties of these four genetic systems offers some explanation for the disproportion in observed frequencies.

\section{System $I$}

Males transmit only the maternal genome (male haploidy). Little need be said here in addition to the above presentation. For purposes of comparison to the other three systems, male haploidy has (i) several means of arising, because it can occur as parthenogenesis or post-fertilisation loss of chromosomes; is (ii) evolutionarily stable, because it is sexual (involves recombination) with a sex ratio equilibrium of $1 / 2$, and it does not readily revert to diploidy; and is (iii) readily discovered in some species because of the parthenogenesis and unusual cytological properties of males. Most of these 
are self-evident, except perhaps for (ii). Discussions of the evolutionary stability of male haploidy are found in Hartl (1972) and Maynard Smith (1978), the latter considering a broad comparison of sexual and asexual reproduction as well.

\section{System II}

Females transmit only the paternal genome (unknown). This shares many of the dynamical evolutionary properties of male haploidy, because the two systems differ only by a change of the sexes (leading to mathematical equivalence). A biological difference is that II can occur only by postfertilisation elimination of chromosomes. In particular, the sperm genome must engineer the elimination of maternal genes from ova. Whether such mutations occur is unknown, but they must also be completely invulnerable to genes which act maternally to restore diploidy. For this single reason, both (i) the origin and (ii) evolutionary stability are questionable. (iii) an observational bias renders this system unlikely to be detected, even if it exists. In II, only the female is unusual and perhaps only in ovogenesis, where detection without markers will be difficult because ovogenesis normally eliminates one set of chromosomes.

\section{System III}

Males transmit only the paternal genome (unknown). The (i) origin of this system requires mutations which cause post-fertilisation loss of the maternal genome, which are in doubt (as in II). The (ii) evolutionary stability of this is even more in question than in II. System III has the additional complication that there is no recombination in the male line, and the female line is continually purged by genes from males. Such populations might be of short lifespan because of the lack of recombination (Maynard Smith, 1978) and because there is selection on the sex ratio to eliminate females. Kin mating, however, can favour the production of daughters and the sex ratio of such a population could vary from all males to nearly all females. (All sex ratio considerations follow from Hamilton's 1967 treatment of $r$-linked sex ratio control.) (iii) Detection of III is facilitated by unusual sex ratios add cytological properties of males.

Males transmit only the paternal genome to sons in some populations of the European frog Rana esculenta, but these males rely on matings with females of a sympatric species for eggs (Uzzell et al., 1975, 1977). R. esculenta probably arose as a two-species hybrid (Uzzell and Berger, 1975; Uzzell, 1978), and it is doubtful that it ever existed in populations resembling system III.

\section{System IV}

Females transmit only the maternal genome (known). This system has seemingly fewer disadvantages than II and III. The (i) origin parallels that for male haploidy because the maternal genome is transmitted uniparentally, so the system may arise parthenogenetically or with post-fertilisation chromosome loss. The (ii) evolutionary stability is problematic because IV is asexual. Selection favours elimination of males under outbreeding ( $\mathrm{cf}$. Maynard Smith, 1978, p. 41), but this will not lead to extinction if daughters can be produced parthenogenetically or if males of neighbouring species can be utilised. Kin mating may favour the production of some sons, but the sex 
ratio should always favour females. The (iii) discovery of these is therefore facilitated by the biased sex ratio and parthenogenesis. However, because of sex ratio selection this system will often be transformed into an all-female population.

There are many species in which daughters transmit only the maternal genome, but most do not produce males in the population. Either they reproduce without males (parthenogenesis) or they use males of sympatric species for sperm (gynogenesis, hybridogenesis; White, 1973; Schultz, 1977; Maynard Smith, 1978). It seems unlikely that many of these evolved through a type IV intermediate with two sexes, because the genetic changes which lead to all-female reproduction often bypass male production at the outset (e.g., suppression of meiosis). There is one example of system IV, however, found in the nematode Rhabditis monohystera (Belar, 1924). The sex ratio in this species is 15 females to one male. Ova are of two types depending upon whether the second meiosis is normal or suppressed. If meiosis is normal, then fertilisation is also normal, and the sperm nucleus fuses with the egg nucleus to give a male. If meiosis II is suppressed, the sperm nucleus degenerates upon entering the egg, and the individual becomes a diploid female. Thus, although the female is diploid, she inherits and transmits only the maternal genome as in IV. The heavily female-biased sex ratio is expected, but it would be interesting to know if mating involves close relatives. Nigon (1949) studied $R$. belari, a species similar or identical to $R$. monohystera. Nigon found that about half of the mictic eggs became female, indicating that the male is probably heterogametic, although chromosome numbers were identical in both sexes. This is now a slight variation of IV, due to the lcw proportion of females which arise from mictic eggs (perhaps an unavoidable consequence of male heterogamety). Pseudogamy is known in several other rhabditids, including some hermaphrodites, but none of these are known to so closely exemplify IV as does $R$. monohystera (Hertwig, 1920; Triantaphyllou, 1976).

In summarising the discussion of the four genetic systems, I and IV seem the most likely to arise and withstand the reversion to diploidy because both can occur parthenogenetically or with maternal control of paternal genome loss. Systems II and III require the possibly rare and vulnerable mutations which cause maternal genome loss. Once arisen, male haploidy (I) evolves much like sexual diploid species, but system IV is asexual and may be short lived (cf. Williams, 1975; Maynard Smith, 1978). Even prior to extinction, IV may eliminate males and become entirely female. Thus, it is not wholly surprising that of these four genetic systems with two sexes, male haploidy is the one most recognised. However, it may be that examples of the others will be discovered when sought from this perspective.

The major conclusion of this paper is that a variety of unusual genetic systems (male haploidy) have a common selective principle that may have guided their evolution. Once recognised, it is seen that this principle applies to a variety of other genetic systems as well. While this principle is not the only factor affecting the evolution of these various systems (or all four systems above would be equally common), it may constitute one of the few advantages for the origin of these systems.

Acknowledgments. - I thank J. F. Grow, R. Lande, K. Aoki, R. Berg, G. C. Williams and E. L. Charnov for comments on the manuscript, R. Kreber, W. Engels, R. J. Schultz, 
W. Moore, T. Uzzell and J. H. Oliver for references, and A. Bull and C. W. Cotterman for translating references. $\mathrm{K}$. Aoki provided a valuable insight for the derivations in the Appendix. C. Hughes prepared the final versions of the illustrations. The author was supported by an NIH Postdoctoral Training Grant Fellowship. This is paper No. 2353 from the Laboratory of Genetics, University of Wisconsin-Madison.

\section{REFERENCES}

BAKER, B. S., AND HALL, J. C. 1976. Meiotic mutants: Genetic control of meiotic recombination and chromosome segregation. In The Genetics and Biology of Drosophila, Vol. la, eds. M. Ashburner and E. Novitski. Academic Press, New York.

BELAR, K. 1924. Die cytologie der merospermie bei freilebenden Rhabditis-arten. Z . Zellen -u. Gewebelehre, 1, 1-24.

BROWN, s. w. 1964. Automatic frequency response in the evolution of male haploidy and other coccid chromosome systems. Genetics, 49, 797-817.

BULl, J. J., AND Charnov, E. L. 1977. Changes in the heterogametic mechanism of sex determination. Heredity, 39, 1-14.

CHARLESWORTH, B., AND HARTL, D. L. 1978. Population dynamics of the segregation distorter polymorphism in Drosophila melanogaster. Genetics, 89, 171-192.

haldane, J. B. s. 1957. Sex determination in Metazoa. Proc. Zool. Soc. Calcutta, Mookerjee Memor. Vol., 13-28.

hall, J. C., Gelbart, W. M., AND Kankel, D. R. 1976. Mosaic systems. In The Genetics and Biology of Drosophila, Vol, la, eds. M. Ashburner and E. Novitski. Academic Press, New York.

hamilton, w. D. 1967. Extraordinary sex ratios. Science, 156, 477-488.

HARTL, D. L. 1972. A fundamental theorem of natural selection for sex linkage or arrhenotoky. Am. Nat., 106, 516-524.

HARTL, D. L., AND BROWN, S. W. 1970. The origin of male haploid genetic systems and their expected sex ratio. Theor. Pop. Biol., 1, 165-190.

haRTL, D. L., AND hiraizumi, y. 1976. Segregation distortion. In The Genetics and Biology of Drosophila, Vol. 1b, eds. M. Ashburner and E. Novitski. Academic Press, New York.

HERTWIG, P. 1920. Abweichende form der parthongenese bei einem mutation von Rhabditis pellio. Arch.f. mikroskop. Anat., 94, 303-337.

hUGHEs-schrader, s. 1948. Cytology of coccids (Coccoidea-Homoptera). Adv. Genet., 2, 127-203.

KERR, w. E. 1974. Advances in cytology and genetics of bees. Ann. Rev. Entomology, 19, 253-268.

Leviran, M. 1963. A maternal factor which breaks paternal chromosomes. Nature, 200, 437-438.

LEVITAN, M., AND williamson, D. L. 1965. Evidence for the cytoplasmic and possibly episomal nature of a chromosome breaker. Genetics, 52, 456.

MAYNARD SMITH, J. 1971. The origin and maintenance of sex. In Group Selection, ed. G. C. Williams. Aldine-Atherton, Chicago.

maynard smith, J. 1978. The Evolution of Sex. Cambridge University Press, Cambridge, UK.

METz, c. w. 1938. Chromosome behavior, inheritance, and sex determination in Sciara. Am. Nat., 72, 485-520.

Nigon, v. 1949. Modalités de la reproduction et déterminisme du sexe chez quelques nématodes libres. Ann. Sci. Nat. (Zool.), 11, 1-132.

NUR, U. 1971. Parthenogenesis in coccids (Homoptera). Amer. Zool., 11, 301-308.

oliver, J. H., JR. 1971. Parthenogenesis in mites and ticks (Arachnida : Acari). Amer. Zool., 11, 283-299.

oliver, J. H., JR. 1977. Cytogenetics of mites and ticks. Ann. Rev. Entomology, 22, 407-429. RICK, C. M. 1971. The tomatoe $\mathrm{Ge}$ locus: linkage relations and geographic distribution of alleles. Genetics, 67, 75-85.

SCHRAder, F., AND hUGHes-SGHRADer, s. 1931. Haploidy in Metazoa. Quart. Rev. Biol., 6, $411-438$.

SCHULrz, R. J. 1977. Evolution and ecology of unisexual fish. Evol. Biol., 10, 277-331.

SONNENBLicK, B. P. 1950. The early embryology of Drosophila melanogaster. In Biology of Drosophila, ed. M. Demerec. Wiley and Sons, New York.

TURNER, B. C. 1977. Resistance to spore killer genes in Neurospora strains from nature. Genetics, 86, 365-366 (Abstr.). 
TURNer, B. C., AND PERkins, D. D. 1976. Spore killer genes in Neurospora. Genetics, 83, 577 (Abstr.).

triantaphyllou, A. c. 1976. Genetics and cytology. Adv. Parasit., 14, 1-34.

UZZELL, T. 1978. Immunological distances between the serum albumins of Rana ridibunda and Rana lessonae. Proc. Acad. Nat. Sci. Phil., 130, 1-10.

UZZELL, T., AND BERGER, L. 1975. Electrophoretic phenotypes of Rana ridibunda, Rana lessonae, and their hybridogenetic associate, Rana esculenta. Proc. Acad. Nat. Sci. Phil., 127, 13-24.

UZZELL, T., BERGER, L., AND GÜNTHER, R. 1975. Diploid and triploid progeny from a diploid female of Rana esculenta (Amphibia : Salientia). Proc. Acad. Nat. Sci. Phil., 127, 81-91. UZZELL, T., GÜNTHER, R., AND BERGER, L. 1977. Rana ridibunda and Rana esculenta: A leaky hybridogenetic system (Amphibia : Salientia). Proc. Acad. Nat. Sci. Phil., 128, 147-171. white, м. J. D. 1954. Animal Cytology and Evolution, 2nd Ed. Cambridge University Press, Cambridge, U.K.

white, м. J. D. 1973. Animal Cytology and Evolution, 3rd Ed. Cambridge University Press, Cambridge, UK.

whiting, P. w. 1945. The evolution of male haploidy. Quart. Rev. Biol., 20, 231-260.

williams, G. c. 1975. Sex and Evolution. Princeton University Press, Princeton, New Jersey. YANAGISAWA, K., DUNN, L. C., AND BENNETT, D. 1961. On the mechanism of abnormal transmission ratios of $\mathrm{T}$ locus in the house mouse. Genetics, 46, 1635-1644.

\section{Appendix \\ Analyses of models (1), (2), and (3)}

Model (1) produces the following recurrence relations

$$
x^{\prime}=\frac{0.5 y(1+x)}{y+s(1-y)}, \quad y^{\prime}=\frac{x+y}{2}
$$

Equation (1a) indicates that for $s=1 / 2$, all values of $(x, y)$ with $x=y$ are stationary. Here we show that there is global convergence to this set from all $x \neq y, 0 \leqq x, y \leqq 1$. It is easily seen that

$$
x^{\prime}-y^{\prime}=\frac{1}{2} \frac{(1-y)}{(1+y)}(y-x) \leqq \frac{1}{2}|x-y|
$$

Thus, the value of $(x, y)$ converges geometrically and rapidly to the line $y=x$, oscillating about $y=x$ each generation. In order to demonstrate that this convergence occurs for all finite intervals of the line $y=x$, we note that $|\Delta y|=\frac{1}{2}\left|x_{0}-y_{0}\right|$. This is the distance that $y$ changes in the first generation from $\left(x_{0}, y_{0}\right)$. The distance that $y$ changes in the second generation is

Thus

$$
\left|\Delta^{(2)} y\right|=\frac{1}{2}\left|x^{\prime}-y^{\prime}\right|=\frac{1}{4} \frac{\left(1-y_{0}\right)}{\left(1+y_{0}\right)}\left|x_{0}-y_{0}\right| \leqq\left(\frac{1}{2}\right)^{2}\left|x_{0}-y_{0}\right| .
$$

$$
\left|\Delta^{(n)} y\right| \leqq\left(\frac{1}{2}\right)^{n}\left|x_{0}-y_{0}\right| .
$$

The farthest that $y$ can move from its initial value $y_{0}$ is therefore

$$
\left|x_{0}-y_{0}\right| \sum_{n=1}^{\infty}\left(\frac{1}{2}\right)^{n}=\left|x_{0}-y_{0}\right| .
$$

By choosing $x_{0}$ and $y_{0}$ sufficiently close to some point $(a, a), 0<a<1$, we can guarantee convergence of $(x, y)$ to within $\pm\left|x_{0}-y_{0}\right|$ vertical distance of $a$, as required.

If $s \neq 1 / 2$, then only two equilibria exist and the equilibrium approached is independent of initial conditions because stability is global. If $s>1 / 2$ then 
$(0,0)$ is stable, while if $s<1 / 2$ then $(1,1)$ is stable. The proofs of global convergence are lengthy and only the outline is considered here; since the proofs in both cases parallel each other, only the case when $s>1 / 2$ is presented. The proof is facilitated by considering a geometrical interpretation (fig. A.l). The set of permissible gene frequencies $(x, y)$ is $0 \leqq x, y \leqq 1$, a unit square.

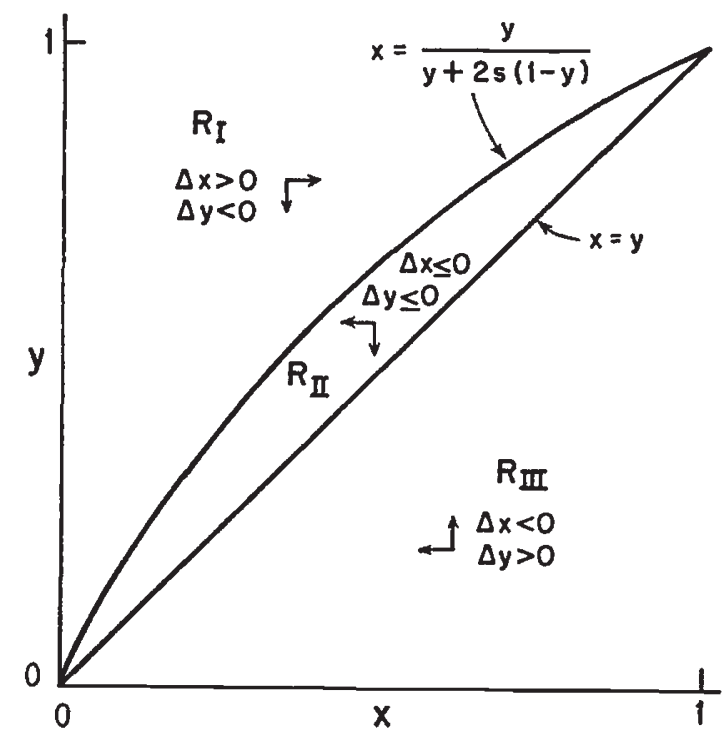

FIG. A.1.-Gene frequency set for model (1). $R_{I}, R_{I I}$, and $R_{I I I}$ define regions in which the trajectory of gene frequency change is within a particular quadrant. Convergence is to $(0,0)$, as discussed in the Appendix. (Drawn for $s=1$.)

This square is subdivided by the functions for which $\Delta x=0$ and $\Delta y=0$ (fig. A.1). These two functions divide the square into three regions:

$$
\begin{aligned}
R_{I}:= & \Delta x>0, \Delta y<0, \text { formed by the union of } x<\frac{y}{y+2 s(1-y)}, \\
& x=0, y=1, \text { but excluding }(0,0) \text { and }(1,1) . \\
R_{I I}:= & \Delta x \leqq 0, \Delta y \leqq 0, \text { formed by the intersection of } \\
& x \geqq \frac{y}{y+2 s(1-y)}, x \leqq y, \text { excluding }(0,0) \text { and }(1,1) . \\
R_{I I}:= & \Delta x<0, \Delta y>0, \text { formed by the union of } x>y, x=1, y=0, \\
& \text { excluding }(0,0),(1,1) .
\end{aligned}
$$

Global convergence can be demonstrated in the following way.

(1) If $(x, y) \in R_{I I}$, then $\left(x^{\prime}, y^{\prime}\right) \in R_{I I}$. In $R_{I I}, \Delta x$ and $\Delta y$ are non-positive, but both are never zero simultaneously nor are both vanishing simultaneously except in approaching equilibrium. The local stability analyses of these two equilibria show that $(0,0)$ is stable but $(1,1)$ is unstable, so convergence to $(0,0)$ is guaranteed whenever $(x, y)$ fall in $R_{I I}$.

(2) If $(x, y) \in R_{I}$, then $\left(x^{\prime}, y^{\prime}\right) \in R_{I I} \cup R_{I I I}$. (If $\left.s \geqq 1,\left(x^{\prime}, y^{\prime}\right) \in R_{I I}\right)$.

(3) If $(x, y) \in R_{I I I}$, then $\left(x^{\prime}, y^{\prime}\right) \in R_{I I} \cup R_{I}$.

(4) For $1 / 2<s<1$, if oscillation occurs so that $(x, y)$ and $\left(x^{\prime \prime}, y^{\prime \prime}\right) \in R_{I}$, 
then $y^{\prime \prime}=y-k$, where $k$ is positive and non-vanishing except near $(0,0)$ and $(1,1)$. Thus, $y$ must converge to zero, even if $(x, y)$ never enters $R_{I I}$. Since $y>x$ in $R_{I}, x$ must also approach zero in $R_{I}$. Limitations on $y^{\prime}$ and $x^{\prime}-y^{\prime}$ in $R_{I I I}$ indicate that $(x, y)$ also approaches zero when in $R_{I I I}$.

Together these four points demonstrate that there is no possible behaviour of $(x, y)$ which is non-convergent to $(0,0)$ for $s>1 / 2$.

Model (2) is similar to model (1). The recursion formulae are

$$
x^{\prime}=\frac{s y+x y(1-s)}{s+x y(1-s)}, \quad y^{\prime}=\frac{x+y}{2}
$$

If $s=1$, then the gene frequencies converge to the set $x=y$, which can be demonstrated as above. If $s \neq 1$, then only two equilibria exist, $(0,0)$ and $(1,1)$. The point $(0,0)$ is locally stable if $s>1$ and is locally unstable if $s<1$. The point $(1,1)$ is locally stable if $s<1$ and is locally unstable if $s>1$. Proof of global convergence was not obtained. Numerical iteration showed that gene frequencies converged toward $(0,0)$ if $s>1$, or to $(1,1)$ if $s<1$ for all initial frequencies tested.

Model (3) leads to the recursion formulae

$$
x^{\prime}=\frac{s y(1-x)+(x / 2)(1+y)}{x+s(1-x)}, \quad y^{\prime}=\frac{x+y}{2}
$$

Only two equilibria occur, $(0,0)$ and $(1,1)$, and the former is locally unstable and the latter locally stable for any positive value of s. Again global convergence was not proved, but numerical iterations showed convergence toward $(1,1)$ for all initial frequencies tested and for all $s$ tested (up to $s=10^{4}$. 